\title{
Typ-2-Diabetes
}

\section{Halbierte Inzidenz bei erhöhtem Verzehr von Olivenöl oder Nüssen}

Salas-Salvado J, Bullo M, Babio N et al. Reduction in the incidence of type 2 diabetes with the Mediterranean diet: results of the PREDIMED-Reus nutrition intervention randomized trial. Diabetes Care 2011;34(1):14-9.

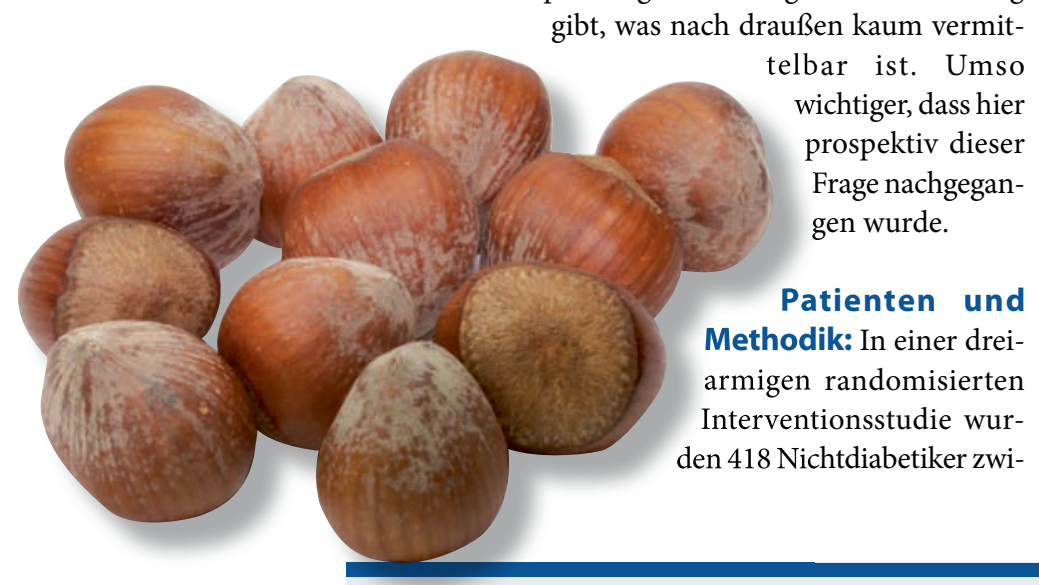

Kommentar: Dies ist eine interessante Studie. Meist wird bisher argumentiert, dass eine mediterrane Diät gut ist. Trotzdem ist die Prävalenz des Typ-2-Diabetes im Mittelmeerraum höher als in Mitteleuropa. Man macht dafür verantwortlich, dass eben zuviel Essen nach Maßgabe einer mediterranen Diät letztendlich auch kontraproduktiv ist. Diese hier vorliegende Studie zeigt nun, dass die konsequente Supplementierung einer mediterranen Diät mit Nüssen oder Olivenöl sehr effektiv die Diabetesinzidenz senken kann. Mehr als die Hälfte des Diabetes konnte durch diese Ernährungsempfehlung verhindert werden. Das ist ähnlich imposant wie in den großen bekannten Präventionsstudien und sollte auch diese Beachtung erfahren. Die Frage, die sich mir allerdings stellt, ist die Nachhaltigkeit. In den Präventionsstudien haben wir gelernt, dass körperliche schen 55 und 80 Jahren in Nordspanien rekrutiert. Die Teilnehmer wurden zufällig in eine Niedrigfetternährungsgruppe, die auch als Kontrollgruppe fungierte, randomisiert, als auch in zwei Gruppen mit mediterraner Diät, wobei die eine Gruppe einen Liter freies Olivenöl pro Woche zusetzen sollte. Die andere Gruppe sollte das gleichermaßen mit $30 \mathrm{~g}$ Nüssen pro Tag tun. Interessanterweise waren die Diätformen ad libitum empfohlen, ohne weitere Empfehlung für körperliche Aktivität. Das Zielkriterium war die Diagnose eines Diabetes mellitus Typ 2 nach Standardkriterien der Amerikanischen Diabetesgesellschaft (ADA).

Ergebnisse: Nach einem mittleren Verlauf von vier Jahren ergab sich eine Diabetesinzidenz von 10,1\% in der Olivenölgruppe, $11 \%$ in der Nussgruppe und 17,9\% in der Low-Fat-Diet-Gruppe. Das multivariat adjustierte Diabetesrisiko war 0,49 und 0,48 in der Olivenöl- bzw. Nussgruppe. Wenn dann beide Gruppen zusammengefasst waren, ergab sich eine Reduktion der Diabetesinzidenz durch eine mediterrane Diät von 52\%. Weiterhin ergab sich diese Diabetesreduktion unabhängig von Veränderungen des Körpergewichts oder körperlicher Aktivität.

Schlussfolgerung: Mediterrane Diät ohne Kalorienrestriktion ist eine effektive Maßnahme in der Prävention des Typ-2-Diabetes bei Personen mit erhöhtem kardiovaskulären Risiko.
Aktivität ein entscheidender Trigger für Nachhaltigkeit ist. Die mediterrane Diät kann zwar signifikant die Diabetesinzidenz senken, aber wenn man sie wieder verlässt (Wash out) kommt auch die Diabetesproblematik zurück, sodass mediterrane Diät einen ähnlichen Effekt wie Medikamente in der Diabetesprävention haben. Sollte das stimmen, ist es trotz alledem nicht weniger wichtig, da die mediterrane Diät mit Olivenöl oder dem vermehrten Verzehr von Nüssen, natürlich mit einem gesünderen Lebensstil einher geht, was zu unterstützen ist.

Prof. Dr. med. Peter E. H. Schwarz

Universität Dresden

Fetscherstr. 74, D-01307 Dresden

Peter.Schwarz@uniklinikum-dresden.de 